\title{
Identifying Interactions that Determine Fragment Binding at Protein Hotspots
}

Chris J. Radoux, Tjelvar S. G. Olsson, Will R. Pitt, Colin R. Groom, Tom L. Blundell

\section{Contents}

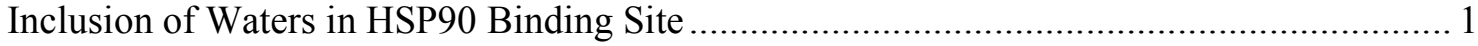

Possible interference of ITC compounds for Pantothenate Synthetase .............................. 2

\section{Inclusion of Waters in HSP90 Binding Site}

Here 2 water molecules known to be important for binding to HSP90 have been included in the calculation of the fragment hotspot maps. Importantly, the waters were protonated using the apo structure and optimisation of the hydrogen bonding network by protoss results in the hydrogens being placed correctly for ligand binding. The donor (blue) map has been contoured at a lower score of 10 in order to include the region resulting from the added water molecule. 


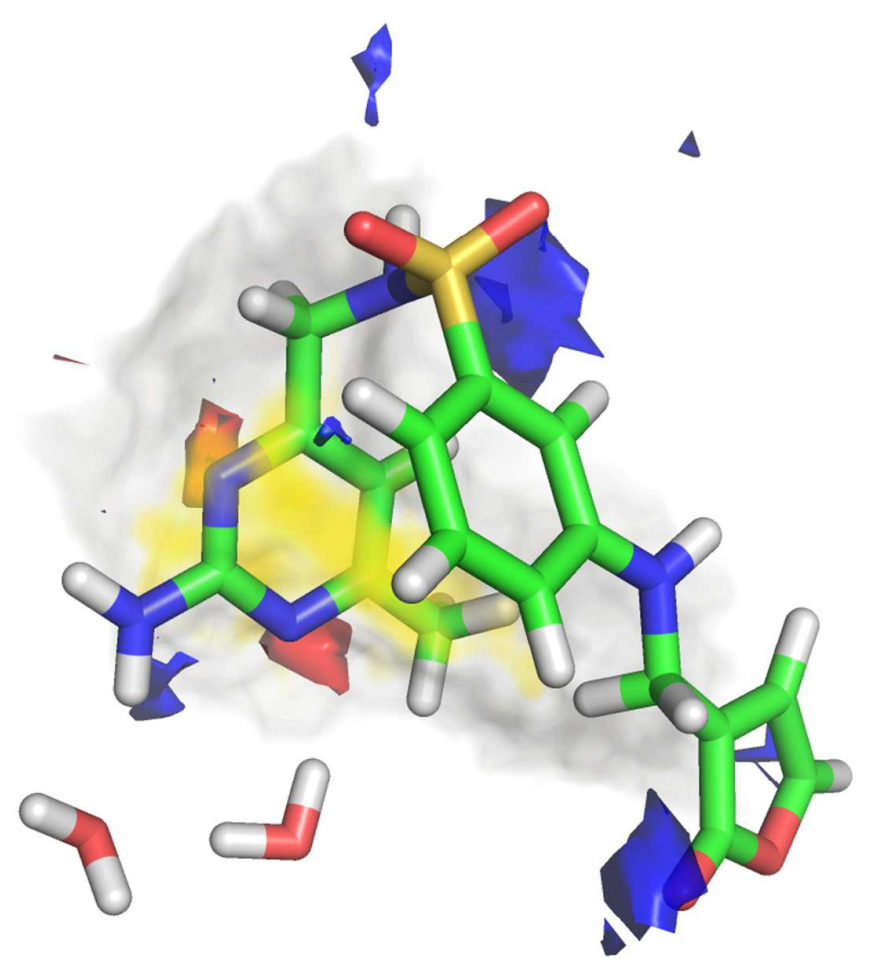

Figure SI1: HSP90 lead (PDB code 2QG0) displayed with maps calculated from apo structure with PDB code 1YES, including two bridging water molecules. The protein has been removed for clarity

\section{Possible interference of ITC compounds for Pantothenate Synthetase}

The ITC experiments contain 4-(2-hydroxyethyl)-1-piperazineethanesulfonic acid (HEPES), which could potentially bind along with the fragment and bridge the interaction. figure SI1 shows a low energy conformer of HEPES aligned such that the sulfonic acid moiety is aligned with the sulfate of PDB: 3IMG. In addition to extending towards the P1 pocket, a charged $\mathrm{NH}$ and hydroxyl oxygen of HEPES are placed in regions of matching high propensity. 


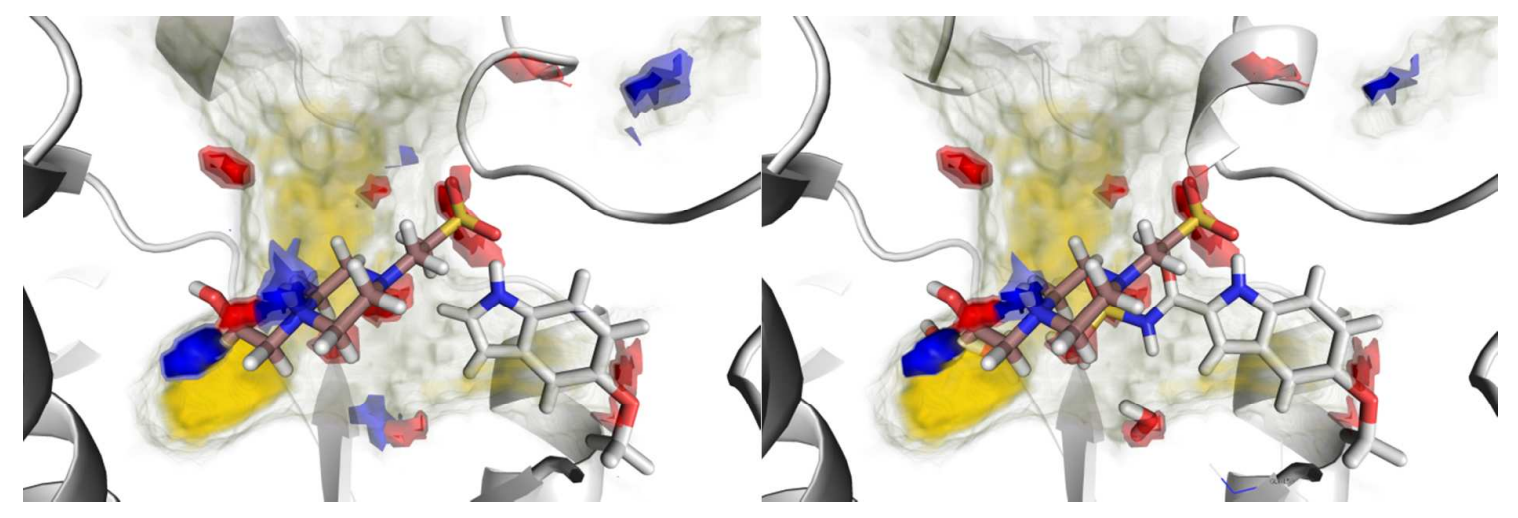

Figure SI2: Structures of 3IMG (left) and 3ISJ (right) with a low energy conformer of HEPES added to replace the sulfate found in the 3IMG structure.

Addition of the sulphonamide moiety shown in figure $5 \mathrm{C}$ has a much lower GE of 0.17 . This is perhaps surprising as one oxygen of the sulfonamide is placed in a very high scoring region of propensity, and the $\mathrm{NH}$ forms a hydrogen bond with a bridging water molecule interacting with Gly-158. This larger molecule would clash with a molecule of HEPES; therefore it is possible that the low GE of this moiety is due to HEPES no longer binding and the indole NH being left unsatisfied.

Although unable to match both oxygen atoms in the same manner as sulfate or HEPES, a water or DMSO molecule may also be capable of bridging the interaction. The crystal structure of 3ISJ does not contain a sulfate, but the nearby crystallographic waters do not bridge the interaction. 